\title{
Silicon nanophotonic wire structures fabricated by 193nm optical lithography
}

\author{
Shankar Kumar Selvaraja ${ }^{1}$, Patrick Jaenen ${ }^{2}$, Stephan Beckx ${ }^{2}$, Wim Bogaert ${ }^{1}$, Pieter Dumon ${ }^{1}$ \\ Dries Van Thourout ${ }^{1}$, Roel Bates ${ }^{1}$ \\ ${ }^{1}$ Photonics research group, University of Gent- IMEC, Gent, Belgium \\ ${ }^{2}$ IMEC, Silicon process technology, Leuven, Belgium
}

\begin{abstract}
We demonstrate the use of $193 \mathrm{~nm}$ optical lithography for fabricating nanophotonic wire structures on silicon-on-insulator (SOI) technology. We present fabrication and measurement result on wire devices. We report a propagation loss of $2.8 \mathrm{~dB} / \mathrm{cm}$ for $450 \times 220 \mathrm{~nm}$ photonic wire.
\end{abstract}

\section{INTRODUCTION}

Much of the photonic research revolves around E-beam lithography for fabrication photonic circuits on SOI technology. With localized optimization e-beam can be used to fabricate high performance devices but it cannot be applied for mass production of such devices. The basic problem with e-beam is the speed, very complex optimization and no standard design rules. With the increase in device size e-beam run into problem of stitching and accuracy errors. DUV (248) optical lithography can over come all these cons with out compromising device performance. We have already demonstrated the use of DUV (248nm) optical lithography for fabricating various photonic devices with high performance $[1,2]$.

With $193 \mathrm{~nm}$ optical lithography we try to demonstrate the effect of high resolution and throughput for mass production in a CMOS fabrication facility. In this paper we present our preliminary results on photonic wire structures fabricated with $193 \mathrm{~nm}$ optical lithography.

\section{FABRICATION PHOTONIC CIRCUIT}

The CMOS foundry facility at IMEC in Leuven, Belgium is utilized to realize the photonic circuits. We use $200 \mathrm{~mm}$ wafer scale technology for all of our experiments. The photonic circuits are fabricated on SOI wafers with $2 \mu \mathrm{m}$ of buried oxide and 220nm of top silicon purchased from SOITEC.

For transferring the pattern from reticle/mask to the wafers we use ASML PAS5500/1100 193nm (dry) scanner. The scanner use ArF laser (193) to illuminate the wafer with the reticle image. Fig. 1 depicts the fabrication process overview. The SOI wafer is first spin coated with bottom antireflective coating (BARC) followed by photoresist. We use chemically amplified photoresist which requires no delay in processing after exposure. Therefore resist process is done on an automated track which is attached to the scanner. After exposure the wafers are baked and developed in the track. The patterned resist is used as an etch mask for silicon etch.

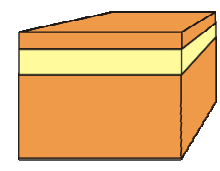

(a) SOI wafer

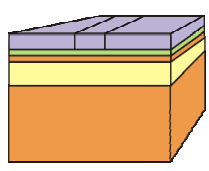

(d) Exposure

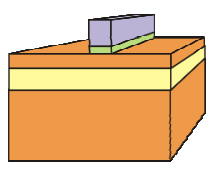

(g) Bottom AR coating etch

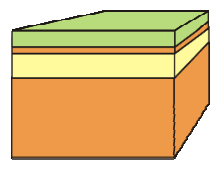

(b) Bottom AR coating and bake

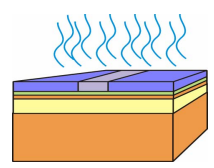

(e) Post-exposure bake

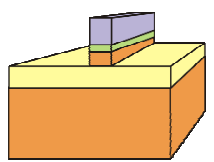

(h) Silicon etch

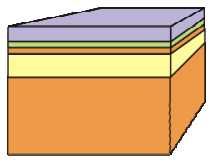

(c) Resist coating and bake

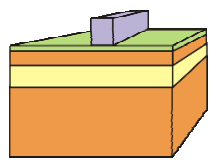

(f) Development

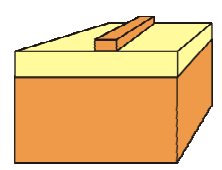

(i) Resist and Bottom AR strip
Figure 1 Fabrication process flow

We use ICP-Dry etching to transfer the photoresist pattern into silicon. Lam research Domino is used for dry etching. We use bottom antireflective coating which is insoluble in water and developer solution. Therefore we used $\mathrm{HBr} / \mathrm{O}_{2}$ chemistry to etch BARC etch. To etch Silicon we use a combination of $\mathrm{HBr} / \mathrm{Cl} 2 / \mathrm{CF}_{4} / \mathrm{He}$ in three different steps and process condition to achieve profile and dimension control. After etching the wafers the remaining photoresist and BARC is removed by combination of dry and wet etch process.

\section{EXPERIMENTAL RESULTS}

We have fabricated photonic wires based devices with $193 \mathrm{~nm}$ lithography and dry etching. Figure 2 depicts the cross section of a photonic wire fabricated with such process. We have fabricated ring resonators and Mach-Zehnder interferometers (fig.3) which are basic wire devices in photonic circuit.

Even though ring resonators are isolated wire based device the coupling region is a very narrow trench $(100-20 \mathrm{~nm})$, in our case it is $180 \mathrm{~nm}$. The effect of lithography and etch process is optimized to get the trench width on target. 


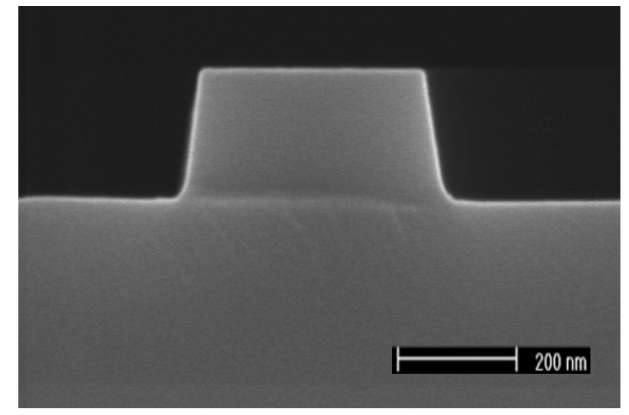

Figure 2 Electron micrograph cross-section of a photonic wire

The etching process in narrow trench is different from the isolated wire structures. Therefore we used different sequence of etching silicon till buried oxide and to make trenches with vertical sidewall. (a)

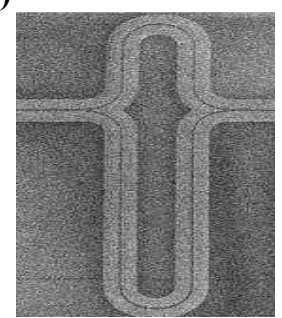

(b)

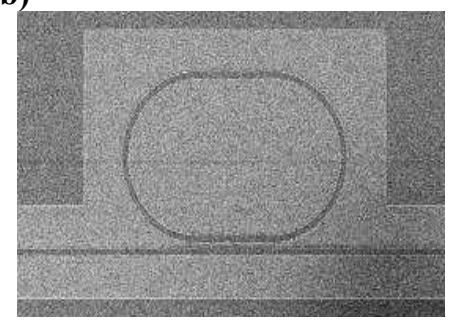

Figure 3 Electron micrograph of (a) MZI and (b) Race track ring resonator

\section{MEASUREMENT RESULTS}

The fabricated photonic wire based components were characterized between the wavelength range of $1500 \mathrm{~nm}$ and $1580 \mathrm{~nm}$.. The light from tunable light source is coupled in and the output is measured with a photodetector. Grating couplers were used to in and out couple light [3].

The propagation loss of the photonic wire was measured with different length of waveguides. To make long wires in a small area spiral wire with large bend radius $(5 \mu \mathrm{m})$ were used. For a bending radius of $5 \mu \mathrm{m}$ the bend loss is very low that the major loss contribution is from the straight section. The lengths of the wires were varied from $0.24 \mathrm{~cm}$ to $5 \mathrm{~cm}$. The propagation loss of a $450 \mathrm{~nm}$ wide photonic wire was measured as $2.8 \mathrm{~dB} / \mathrm{cm} \pm 0.28 \mathrm{~dB} / \mathrm{cm}$ around $1550 \mathrm{~nm}$ for TE polarization. The propagation loss is comparable to the best result found in the literature, without any post processing like sidewall smoothing.

Ring resonators are used in wide spectrum of integrated optical functionalities; some of them are wavelength filters, add-drop multiplexers and optical sensors. Most of these applications require high $\mathrm{Q}$ and finesse. All pass ring resonator with ring radius of $4 \mu \mathrm{m}$ and coupling gap of $180 \mathrm{~nm}$ was fabricated as the basic test device. All pass ring resonators are interesting for optical delays which can be tailored in to optical buffers in high bit rate optical communication. We measured a Q of 19,500 and finesse of 200 which demonstrated the quality of device fabricated with $193 \mathrm{~nm}$ optical lithography.

MZI's are one of the building blocks for photonic integrated circuits. They can be used as broadband filters, switches or modulators. We fabricated 1X1 MZI's using Y-splitter with delay length of $50 \mu \mathrm{m}$ which results in a FSR of $10 \mathrm{~nm}$. Fig. 4 shows the transmission spectra of MZI. Insertion loss of around $4 \mathrm{~dB}$ was measured which is mainly due to the Ysplitter. With the well balanced splitting we achieved an extinction ratio of $30 \mathrm{~dB}$.

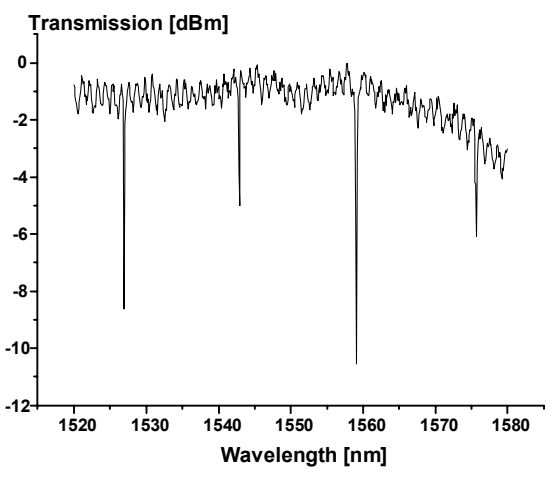

Figure 4 Transmission measurement of all pass ring resonator

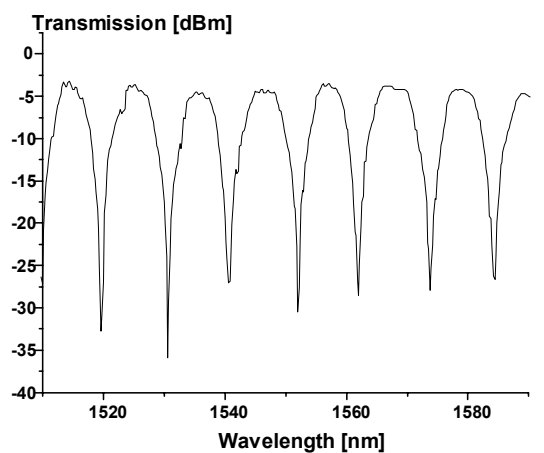

Figure 5 Transmission measurement of a MZI

\section{SUMMARY}

We have demonstrated the use of $193 \mathrm{~nm}$ optical lithography and dry etching for fabricating photonic wire structures. We measured propagation loss as low as $2.8 \mathrm{~dB} / \mathrm{cm}$ for $450 \mathrm{~nm}$ photonic wire. For racetrack all pass ring resonator we measured Q factor of 19,500. The devices presented show the capability of $193 \mathrm{~nm}$ lithography for fabricating photonic devices.

\section{REFERENCE}

[1] P. Dumon et al., "Linear and nonlinear nanophotonic devices based on silicon-on-insulator wire waveguides", Jap. J. of Applied Physics, vol. 45, no.8B, pp. 6589-6602, 2006.

[2] W. Bogaerts et al., "Nanophotonic Waveguides in Silicon-on-Insulator Fabricated with CMOS Technology", J. Lightwavve Technonol., vol. 23, no. 1, pp. 401-412, 2005.

[3] D. Taillaert et al., "An Out-of-Plane Grating Coupler for Efficient ButtCoupling Between Compact Planar Waveguides and Single-Mode Fibers", IEEE J. of Quantum Electron., vol. 38, no. 7, pp.949-955, 2002. 